\title{
Efficient Password-Authenticated Key Exchange for Three-Party Secure Against Undetectable On-Line Dictionary Attacks ${ }^{\star}$
}

\author{
Jeong Ok Kwon ${ }^{1,2}$, Kouichi Sakurai ${ }^{1}$, and Dong Hoon Lee ${ }^{2}$ \\ 1 Department of Computer Science and Communication Engineering, \\ Kyushu University, Japan \\ pitapat@cist.korea.ac.kr, sakurai@csce.kyushu-u.ac.jp \\ 2 Center for Information Security Technologies (CIST), Korea University, Korea \\ donghlee@korea.ac.kr
}

\begin{abstract}
A password-authenticated key exchange (PAKE) protocol in the three-party setting allows two users communicating over a public network to agree on a common session key by the help of a server. In the setting the users do not share a password between themselves, but only with the server. In this paper, we explore the possibility of designing a round-efficient three-party PAKE protocol with a method to protect against undetectable on-line dictionary attacks without using the random oracle. The protocol matches the most efficient three-party PAKE protocol secure against undetectable on-line dictionary attacks among those found in the literature while providing the same level of security. Finally, we indentify the relations between detectable on-line and undetectable on-line dictionary attacks by providing counter-examples to support the observed relations?.
\end{abstract}

Keywords: Cryptography, password-authenticated key exchange, dictionary attacks, round complexity, mobile network security.

\section{Protocols for PAKE in the Three-Party Setting}

We now present our two protocols $3 \mathcal{P} \mathcal{A K \mathcal { E }} 1$ and $3 \mathcal{P} \mathcal{A} \mathcal{K} \mathcal{E} 1$ for PAKE in the three-party setting. $3 \mathcal{P} \mathcal{A} \mathcal{K} \mathcal{E} 1$ is designated to protect passwords from detectable on-line and off-line dictionary attacks and is explicit authentication. $3 \mathcal{P} \mathcal{A K \mathcal { E }} 2$ is designated to enhance $3 \mathcal{P} \mathcal{A} \mathcal{K} \mathcal{E} 1$ against undetectable on-line dictionary attacks in addition to detectable on-line and off-line dictionary attacks, yet still requires four rounds and is implicit authentication. In this paper, we assume the parties can transmit messages simultaneously.

\footnotetext{
* This work was done while the first author visits in Kyushu Univ. and was supported by the Ministry of Information \& Communications, Korea, under the Information Technology Research Center (ITRC) Support Program.

${ }^{1}$ This is a very partial version of the full paper available at http:// cist.korea.ac.kr/pitapat/JeongOkKwonTriS.ps
} 
$3 \mathcal{P} \mathcal{A K E} 1$

Public information. Two primes $p, q$ such that $p=2 q+1$, where $p$ is a safe prime such that the decision Diffie-Hellman problem is hard to solve in $\mathbb{G}$. A finite cyclic group $\mathbb{G}$ has order $q$, and $g_{1}, g_{2}$ are generators of $\mathbb{G}$ both having order $q$, where $g_{1}$ and $g_{2}$ must be generated so that their discrete logarithmic relation cannot be known. A hash function $H$ from $\{0,1\}^{*}$ to $\{0,1\}^{l}$. A message authentication code (MAC), Mac $=$ (Mac.gen, Mac.ver). Given a random key $k$, Mac.gen computes a tag $\tau$ for a message $M$; we write this as $\tau=$ Mac.gen $_{k}(M)$. Mac.ver verifies the message-tag pair using the (shared) key, and returns 1 if the tag is valid or 0 otherwise. $F$ is a pseudo random function family.

Initialization. Each user $U_{i}$ for $i \in\{1,2\}$ obtains $p w_{i}$ at the start of the protocol using a password generation algorithm $\mathcal{P G}\left(1^{k}\right)$ which on input a security parameter $1^{k}$ outputs a password $p w_{i}$ uniformly distributed in a password space password. Then $U_{i}$ sends $v_{i, 1}=g_{1}^{H\left(U_{i}\|S\| p w_{i}\right)} \bmod p$ and $v_{i, 2}=g_{2}^{H\left(U_{i} \| S|| p w_{i}\right)} \bmod p$ which are verifiers of the password to the server $S$ over a secure channel. Upon receiving the verifiers, $S$ stores them in a password file with an entry for $U_{i}$. The indices are cyclic, i.e., $U_{n+1}$ is $U_{1}$, where $n=2$.

Round 1. An initiator $U_{i}$ for $\mathrm{i}=1$ broadcasts $\left(U_{i}, U_{i+1}, S\right)$.

Round 2. Each user $U_{i}$ chooses a random number $x_{i} \in \mathbb{Z}_{q}^{*}$, computes $X_{i, S}=g_{1}^{x_{i}}$. $v_{i, 2} \bmod p$, and sends $\left(U_{i}, X_{i, S}\right)$ to the server.

Round 3. Upon receiving $\left(U_{i}, X_{i, S}\right), S$ selects a random number $s \in \mathbb{Z}_{q}^{*}$ and computes $X_{S, i}=\left(X_{i+1, S} / v_{i+1,2}\right)^{s} \cdot v_{i, 2} \bmod p$, and sends $\left(S, X_{S, i}\right)$ to each user $U_{i}$ for $i=\{1,2\}$.

Round 4. Upon receiving $\left(S, X_{S, i}\right)$, each user $U_{i}$ computes $k_{i}=\left(X_{S, i} / v_{i, 2}\right)^{x_{i}} \bmod p$ and $\tau_{i}=$ Mac.gen $_{k_{i}}\left(U_{i}\left\|U_{i+1}\right\| S\right)$ and sends $\left(U_{i}, \tau_{i}\right)$ to $U_{i+1}$.

Key computation. Upon receiving $\left(U_{i}, \tau_{i}\right)$, each user $U_{i+1}$ computes Mac.ver $_{k_{i+1}}\left(\tau_{i}\right)$. Each user $U_{i+1}$ halts if Mac.ver returns 0 or computes the session key $s k_{i+1}=F_{k_{i+1}}\left(U_{1}\|S\| U_{2}\right)$ otherwise.

Remark. We have observed that the relations between detectable and undetectable on-line dictionary attacks. This is, the security against detectable on-line dictionary attacks does not necessarily imply the security undetectable on-line dictionary attacks and vice versa. To support the observed relations we consider counter-examples presented in this paper. For the first relation, consider our first protocol $3 \mathcal{P} \mathcal{A K} \mathcal{E} 1.3 \mathcal{P} \mathcal{A K} \mathcal{E} 1$ is secure against detectable on-line dictionary attacks but it is insecure against undetectable on-line dictionary attacks. For the second relation, consider our second protocol $3 \mathcal{P} \mathcal{A K \mathcal { E }} 2$. We can modify $3 \mathcal{P} \mathcal{A} \mathcal{K} \mathcal{E} 2$ to secure against undetectable on-line dictionary attacks but to insecure against detectable on-line dictionary attacks. If the MAC verification between each user and the server is removed in Round 4 of $3 \mathcal{P} \mathcal{A K \mathcal { E } 2} 2$, the protocol does not secure against on-line dictionary attacks anymore because the users cannot detect and log the failed guesses. Therefore we must independently consider the both attacks and provide methods to resistant to both attacks when design secure protocol for 3-party PAKE. 
$3 \mathcal{P} \mathcal{A K \mathcal { K } 2}$

Same as $3 \mathcal{P} \mathcal{A} \mathcal{K} \mathcal{E} 1$ except the following points.

Round 2. Each user $U_{i}$ chooses a random number $x_{i} \in \mathbb{Z}_{q}^{*}$, computes $X_{i, S}=g_{1}^{x_{i}} \cdot v_{i, 2} \bmod p$, and sends $\left(U_{i}, X_{i, S}\right)$ to the server $S$. For $i=\{1,2\}$, $S$ chooses random numbers $y_{i} \in \mathbb{Z}_{q}^{*}$, computes $X_{S, i}=g_{1}^{y_{i}} \cdot v_{i, 2} \bmod p$, and sends $\left(S, X_{S, i}\right)$.

Round 3. Upon receiving $\left(S, X_{S, i}\right)$, each user $U_{i}$ computes $k_{i, S}=\left(X_{S, i} / v_{i, 2}\right)^{x_{i}} \bmod p$ and $\tau_{i, S}=$ Mac.gen $_{k_{i, S}}\left(U_{i}\|S\| X_{i, S} \| X_{S, i}\right)$ and sends $\left(U_{i}, \tau_{i, S}\right)$ to $S$.

Round 4. Upon receiving $\left(U_{i}, \tau_{i, S}\right), \quad S$ computes Mac.ver $_{k_{S, i}}\left(\tau_{i, S}\right)$ where $k_{S, i}=\left(X_{i, S} / v_{i, 2}\right)^{y_{i}} \bmod p$ for $i=\{1,2\} . S$ halts if at least one of Mac.ver $k_{S, i}$ returns 0 or proceeds to the next process otherwise. $S$ selects a random number $s \in \mathbb{Z}_{q}^{*}$, and computes $Y_{S, i}=\left(g_{1}^{x_{i+1}}\right)^{s}$ and $\tau_{S, i}=\operatorname{Mac} \operatorname{gen}_{k_{S, i}}\left(U_{i}\left\|U_{i+1}\right\| Y_{S, i}\right)$ and sends $\left(S, Y_{S, i}, \tau_{S, i}\right)$ to each user $U_{i}$.

Key computation. Upon receiving $\left(S, Y_{S, i}, \tau_{S, i}\right)$, each user $U_{i}$ computes $\operatorname{Mac} \operatorname{ver}_{k_{i}}\left(\tau_{S, i}\right)$. Each user $U_{i}$ halts if Mac.ver returns 0 or computes the session key $s k_{i+1}=F_{k_{i+1}}\left(U_{1}\|S\| U_{2}\right)$ otherwise, where $k_{i}=\left(Y_{S, i}\right)^{x_{i}} \bmod p$.

SECURITY OF $3 \mathcal{P} \mathcal{A K} \mathcal{E} 2$.

1. $3 \mathcal{P} \mathcal{A K E} 2$ is secure against detectable on-line dictionary attacks since the users are able to detect a failed guess by the MAC verification in Round 4. $3 \mathcal{P} \mathcal{A K E} 2$ is resistant to undetectable on-line dictionary attacks since the server is able to depart honest requests from malicious attempts. If the MAC verification in Round 3 is failed, the server will notice that whose password to being a target of undetectable on-line dictionary attacks and it be at a crisis. After a small amount of failed guesses the server reacts and informs the target user to stop any further use of the password and to change the password into a new one. $3 \mathcal{P} \mathcal{A} \mathcal{K} \mathcal{E} 2$ is secure against off-line dictionary attacks via the difficulty of the decision DDH problem.

2. Key secrecy means that no computationally bounded adversary should learn anything about session keys shared between two honest users. Especially in the 3-party key exchange model existing a server, more desirable security notion is that the participating server should not learn anything about session keys shared between two honest users, additionally. $3 \mathcal{P} \mathcal{A} \mathcal{K} \mathcal{E} 2$ provides strong key secrecy since passwords are unguessed from dictionary attacks and in order to learn some significant information on session keys, the adversary $\mathcal{A}$ including $\mathcal{S}$ has to solve the $\mathrm{DDH}$ problem or break the MAC which is selectively unforgeable against adaptively chosen message attacks.

3. Forward secrecy means that even if long-term secret keys of one or more users are compromised, the secrecy of previous session keys established by honest users without any interference by the adversary is not affected. $3 \mathcal{P} \mathcal{A K \mathcal { E } 2}$ provides forward secrecy under the DDH assumption. That is, even if the 
passwords of users are compromised, any adversary cannot learn any significant information on previously established session keys under the DDH assumption.

4. A protocol is secure against known key attack (or Denning-Sacco attack) if the following conditions hold. First, even with the session key from an eavesdropped session an adversary cannot gain the ability to impersonate the legitimate user directly. Second, an adversary cannot gain the ability to performing off-line dictionary attacks on the users' passwords from using the compromised session keys which are successfully established between honest entities. Third, a legitimate user $\mathcal{U}_{i}$ knowing $p w_{i}$ does not learn any information about the partner $U_{i+1}$ 's password $p w_{i+1}$ from the session key established with $U_{i+1}$. In $3 \mathcal{P} \mathcal{A} \mathcal{K} \mathcal{E} 2$, the session keys are computationally independent from each other. Thus, a compromised old session key is not helpful for $\mathcal{A}$ in attempting known key attack. To break the second condition $\mathcal{A}$ has to solve the DDH problem. The security on the third condition is also provided under the DDH assumption. That is, to successfully mount an off-line dictionary attack from the session key established with $U_{i+1}, \mathcal{U}_{i}$ knowing $p w_{i}$ has to solve the DDH problem, in which one's password $p w_{i}$ is not helpful in attempting the off-line dictionary attack against $p w_{i+1}$.

\section{Concluding Remarks}

In this paper, we have proposed a three-party PAKE protocol secure against detectable/undetectable on-line and off-line dictionary attacks without making use of the random oracle model. However, we only provided informal security analysis. Proving the security of our protocol in the standard model is the subject of ongoing work. 